\title{
LA ORGANIZACIÓN SOCIAL DE LA REPRODUCCIÓN DE LOS AGENTES SOCIALES, LAS UNIDADES FAMILIARES Y SUS ESTRATEGIAS
}

\author{
CARLOS A. BARSOTTI*
}

\section{INTRODUCCION}

HASTA EL PRESENTE, tal como se ha hecho notar, la organización social de la reproducción de los agentes sociales ha sido imperfectamente integrada a los anảlisis, o no ha sido considerada como un problema fundamental, o ha sido estudiada sólo parcialmente. (Meillassoux, 1979; Singer, s.d., a; Naciones Unidas, 1973). Por sus limitaciones, este artículo no escapará a esas características y, además, su estructura requiere algunas aclaraciones.

La primera sección intenta proponer una aproximación a un marco interpretativo (necesariamente abstracto y general) para el análisis de las diferentes formas de organización social de la reproducción de los agentes sociales, limitándose a los aspectos organizativos y a sus implicaciones culturales y políticas. Se sugiere que dicho marco puede ser susceptible de aplicación a la forma en que está organizada la satisfacción de distintos tipos de necesidades: salud y asistencia médica, vivienda, alimentación, vestuario, educación, transporte, comunicaciones, etc.

La segunda sección está dedicada a una elucidación conceptual de las unidades familiares y las distintas maneras de determinar un referente empirico de las mismas que pueda considerarse como unidad de decisión y de recursos, en relación con la organización social de la reproducción de los agentes sociales.

La tercera sección se refiere a las estrategias familiares en cuanto nexo entre la organización social de la reproducción de los agentes sociales y las unidades familiares responsables de dicha reproducción.

Las continuas y a veces extensas referencias a la educación tienen sólo un carácter ilustrativo; se deben a que el autor ha estado trabajando últimamente sobre el tema y se proponen como sugerencia para el lector más próximo a las formas de satisfacer otros tipos de necesidades, reflexione sobre ellas.

Para el tratamiento de los tres temas enunciados, se tiene en cuenta la situación predominante en América Latina, en lo que respecta a la organiza-

\footnotetext{
* El autor es funcionario del Proyecto UNESCO/CEPAL/PNUD "Desarrollo y Educación en América Latina y el Caribe" pero los juicios vertidos en el artículo no comprometen a las organizaciones indicadas.
} 
ción social de la reproducción de los agentes sociales y, en particular que: a) se trata de sociedades de clases ancladas en un modo de producción capitalista dependiente y periférico, con marcada heterogeneidad estructural (Pinto y Di Filippo, 1979; Di Filippo, 1980); b) una parte importante, variable según los países y las clases sociales, de los bienes y servicios reproductivos, es decir, de aquellos bienes-y servicios destinados al consumo de los individuos y de las unidades familiares, es producida socialmente (en unidades económicas distintas, de las unidades familiares o por éstas pero destinados al mercado) y son adquiridos mediante transacciones monetarias; c) la masa global de esos bienes y servicios es distribuida de acuerdo con reglas que implican una marcada desigualdad.

De estas características generales, se desprenden algunas consecuencias. En primer término, que la unidad mínima de significación es el EstadoNación (Singer, a, s.d.) y que, en consecuencia, la organización social de la reproducción de los agentes sociales queda planteada en términos de agrupamientos de agentes sociales en competencia, bajo condiciones políticas que varían en las distintas sociedades (Giffin, 1980). Esto lleva a reconsiderar y a cuestionar todo el problema del poder en los diversos ámbitos en que se ejerce (la familia, la escuela, la alimentación, el vestuario, la vivienda, etc.) y a poner en evidencia las limitaciones de una concepción de su naturaleza que se restrinja sólo a los aspectos jurídicos. "En el siglo XVIII, una de las grandes novedades en "la técnica del poder fue el surgimiento, como problema económico y político, "de la 'población'... Los gobiernos advierten que no tienen que vérselas con "individuos simplemente, ni siquiera con un 'pueblo', sino con una 'población' "y sus fenómenos específicos, sus variables propias: natalidad, morbilidad, "duración de la vida, fecundidad, estado de salud, frecuencia de las enfermedades, "forma de alimentación y de vivienda" (Foucault, 1978). Por cierto, el poder no utiliza esa técnica sólo en beneficio de la 'población' sino también, y principalmente, en el suyo propio y en los más diversos ámbitos de la vida social.

En efecto, en toda sociedad hay una masa global de bienes y servicios disponibles para el consumo directo de los individuos y de las unidades familiares que se asigna a las distintas clases o sectores sociales según ciertas reglas de distribución que expresan las probabilidades que tienen esas clases de apropiarse ( $y$, por consiguiente, de expropiar a otras clases) de una porción de esos bienes y servicios, de distinta calidad y cantidad. Esas probabilidades de apropiación y expropiación involucran una pugna social y ésta es una forma de hostilidad, aunque no esté acompañada de ningún acto abiertamente hostil, ni de intenciones o sentimientos hostiles percibidos por los participantes en la pugna. Por otro lado, esta pugna alrededor de la masa global de bienes y servicios reproductivos es una forma de relación humana que no consiste en actos aislados aunque se manifieste y se concrete en ellos, y en la cual, quienes participan en ella desde distintas posiciones, condicionan reciprocamente sus acciones. (Clausewitz, 1976). En estas condiciones, la organización social de la reproducción de los agentes sociales puede ser un mecanismo que, al mismo tiempo, concreta los resultados de la pugna social abierta, latente o reprimida y oculta el conflicto. 
En segundo término, se ha señalado que el desarrollo capitalista de una sociedad dada no puede ser analizado y aprehendido críticamente mediante el solo estudio del funcionamiento de las leyes de reproducción del capital, ya que "sus contradicciones adquieren sus rasgos específicos en el plano social y político "en la medida en que se combinan con las contradicciones entre el modo de "reproducción del capital y el de la población. El resultado de las contradicciones "entre el modo como se reproduce el capital, y el modo como se reproduce la población "puede influir profundamente, a su vez, sobre uno y otro modo de reproducción" (Singer, a, s.d., el subrayado es nuestro). Tal como se ha indicado, la sección siguiente se limita a considerar la organización de la reproducción de la población en sus aspectos más generales.

Antes de terminar esta introducción y para entrar en materia se recurrirá a una primera ilustración extraída del campo educativo.

En un estudio sobre la expansión educacional y la estratificación social en América Latina entre 1960 y 1970 (Filgueira, 1977), se sostenía que el retraso escolar estaría condicionado por el origen social de los individuos, la diferenciación según zona de residencia y las características del sistema educacional. Representando en forma dicotómica todas las combinaciones posibles entre esos determinantes, se llegaba a un cuadro de ocho celdas. En dicho estudio se proponía que la primera y la última celda representarian los extremos de mayor y menor incidencia del retraso escolar, respectivamente, correspondiendo las celdas intermedias a un continuo que se extiende entre ambos extremos.

Otro estudio destacaba la utilidad del esquema analítico precedente para organizar la información y hacía notar que, a los efectos interpretativos, era necesario hacer las siguientes precisiones: 1) Las ocho celdas definidas no constituyen realidades aisladas, ya que las dimensiones básicas sobre las cuales se construyen remiten a elementos unificadores: las zonas de residencia son ámbitos de una misma sociedad nacional; los distintos origenes sociales sólo pueden ser comprendidos adecuadamente dentro del sistema de clases de una søciedad nacional; la distinta calidad de aquella parte del sistema educativo a la que se accede tiene sentido si se ubica en el marco del sistema nacional de prestación de ese servicio, así como de la distribución general de ingresos, bienes y servicios. 2) La calidad del sistema eduçativo no puede limitarse sólo al sistema de educación formal, ya que en la socialización de los agentes sociales inciden también una variedad de organizaciones $y$ ' agentes $y$, en especial, las unidades familiares, portadoras de un 'ethos' educativo, de un patrimonio cultural y situadas en una ubicación social que condiciona la distancia a los bienes y equipamientos culturales y a los centros de producción y transmisión de cultura (Borsotti, 1980).

En este estudio se sostenía que los circuitos de satisfacción de necesidades incluyen, por un lado, las responsabilidades de las unidades familiares y sus estrategias para cumplirlas y, por el otro, los mecanismos políticos, administrativos, técnicos y organizativos de prestación de los servicios. En lo que respecta a la educación, se señalaba que las formas socio-organizativas, vigentes van desde las escuelas primarias privadas, con profesores extranjeros 
que enseñan en su idioma, con buenos materiales didácticos y pedagogía activa, hasta las escuelas rurales unitarias, incompletas, con profesores inexpertos y carentes de materiales didácticos; desde las universidades privadas exclusivas y la realización de estudios de post-grado en el extranjero hasta los programas de capacitación para el cuidado de animales domésticos realizados por voluntarios. En consecuencia, se sugerían algunas hipótesis de trabajo de carácter general:

a) La demanda agregada efectiva de servicios educativos que hacen las unidades familiares difiere en calidad y cantidad según su situación de clase y la zona de residencia;

b) En una misma sociedad coexisten distintas formas socio-organizativas de obtener servicios educativos y esas formas son más o menos disímiles según los estilos de desarrollo;

c) Tanto las unidades familiares como el servicio educativo al que acceden, tienen distintas calidades y valores incorporados y estas diferencias habrán de manifestarse en definitiva en las características de los agentes sociales que los insumen;

d) A menos que se modifiquen las condiciones actuales, la reproducción de los agentes sociales a través de las diversas formas socio-organizativas vigentes, conducirá muy probablemente al mantenimiento o el agravamiento de las desigualdades educativas actuales (Borsotti, 1980).

\section{LA ORGANIZACIÓN SOCIAL DE LA REPRODUCCIÓN DE LOS AGENTES SOCIALES}

La reproducción de los agentes sociales incluye dos ciclos: el generacional y el cotidiano.

El ciclo generacional remite a dos grandes esferas:

a) La reproducción biológica: concepción, gestación, nacimiento, vida, muerte y se relaciona con hechos tales como los acoplamientos, las características de las uniones maritales, la fertilidad, la salud, la asistencia médica y los diferentes determinantes, de la longevidad. Los distintos aspectos de la reproducción biológica han sido objeto de mayor atención que aquéllos referidos a la otra esfera de la reproducción generacional, en especial por parte de la demografía;

b) La reproducción psico-cultural. Reproducir cultura no es sólo una cuestión de instrucción fcrmal y de disciplina, lo que podría lograrse sólo por intermedio del sistema educativo formal. También, y fundamentalmente, es cuestión de que la personalidad toda quede embebida de determinada forma de cultura, que el individuo quiera hacer lo que tiene que hacer (Lasch, 1975). La socialización temprana y tardía, la educación formal, la participación en distintas prácticas sociales, no sólo proporcionan la capacitación cognoscitiva para ocupar ciertas posiciones, sino que también generan una disposición interior para poner en práctica las normas, pautas, valores y actitudes referentes a lo económico, lo político, la autoridad en sus, 
distintas manifestaciones, lo emocional, la información, las decisiones, etc. Es decir, todo aquello que convierte al individuo en un agente social.

El ciclo cotidiano remite al mantenimiento de la existencia de los agentes sociales en sus distintos aspectos. Por razones de comodidad y por ser más visible, puede considerarse el consumo de bienes y servicios que, en las encuestas de hogares son normalmente clasificados en los siguientes rubros: alimentación, vestuario, vivienda, equipos y operación de los hogares, transporte, comunicaciones, cuidados personales, recreación, ceremonias y regalos, impuestos, seguros, etc. (Argentina, 1970).

La reproducción de los agentes sociales debe considerarse en relación con las posiciones existentes en la sociedad y con los procesos de selección social a que están sometidos tanto los agentes como las posiciones. Según dichos procesos, algunas posiciones tienden a desaparecer; otras, son relativamente estables; otras, emergentes. Por otra parte, a través de su proceso de reproducción, los agentes sociales incorporan características que les abren distintas probabilidades de acceder a uno u otro tipo de posiciones., El proceso de reproducción, de los agentes sociales es, también, un proceso de selección social. Así, por ejemplo, para la cohorte aparente que comenzó sus estudios formales en 1960 y debió terminarlos en 1977, en la Argentina, se definieron las siguientes categorias de agentes sociales: un 3\% no accedió al sistema escolar; un $52 \%$ no terminó el ciclo primario; un $15 \%$ terminó primaria y no comenzó secundaria; un $26 \%$ comenzó secundaria; un $4 \%$ completó estudios superiores.

Ahora bien, en América Latina, las unidades familiares son responsables de la reproducción de los agentes sociales en el ciclo cotidiano y generacional. De alguna manera, la afirmación precedente encuentra apoyo en los bajos porcentajes de la población que vive en hogares privados unipersonales y colectivos. Este hecho sugiere que la reproducción cotidiana y generacional de la gran mayoría de la población se da ininterrumpidamente en el marco de unidades familiares de orientación y de procreación, dentro de las cuales los individuos realizan sus cursos vitales. Esta responsabilidad de las unidades familiares no implica que ellas ejecuten directamente todas las actividades pertinentes, siendo el caso más frecuente que sólo se hagan cargo de ellas en forma parcial. La fracción de esas actividades que se ejecuta directamente por las unidades familiares y por unidades de otro tipo varia, entre otras cosas, según el grado de desarrollo de los países, y según la clase social y la zona de residencia de las unidades familiares.

La distin ión entre responsabilidad por la reproducción de los agentes sociales y la ejecución directa de las actividades pertinentes ubica a las unidades fámiliares como una de las diversas unidades que participan en la producción de los mismos hombres y, al mismo tiempo, plantea el problema de las otras unidades y de la organización social de todo el proceso de reproducción de los agentes sociales (Le Nouvel Observateur, 1976; Carlos y Sellers, 1972). Dicha distinción permite, al mismo tiempo, aclarar la situación de las unidades familiares como unidades de producción. La reproducción de los agentes sociales en el ciclo cotidiano y generacional (o, lo que es lo mismo, su producción), se da en la esfera de la circulación y distribución de bienes y 
servicios, por lo que aparece como externa al proceso productivo global. Esto ha conducido a considerar a las unidades familiares como unidades de consumo, ocultando su carácter de unidades responsables de la producción de los agentes sociales. Otra fuente de confusión proviene del hecho de que quienes realizan los consumos finales son los individuos. Queda por determinar en qué medida las cantidades y calidades que consumen los individuos se enmarcan en decisiones de las unidades familiares, dentro de lo que les ha correspondido según su pertenencia de clase, de acuerdo a las reglas generales de distribución.

El conjunto de bienes y servicios para la reproducción cotidiana y generacional de los agentes sociales, se manifiesta como necesidades. Ese conjunto de "medios de vida necesarios" es muy variable y flexible y está configurado por todo aquello que "en nuestro país y en nuestros dias, constituye la manutención habitual de un hombre de mi clase" (Luxemburgo, 1972). Desde un punto de vista puramente económico, el nivel de vida (en cuanto canasta de bienes y servicios que componen el consumo de un grupo social) es un dato social y para ello basta que sea reconocido socialmente como minimo de subsistencia, adquiera fijeza institucional y, sobre todo, que sea defendido como tal (Marshall, 1979). Cabe insistir en dos aspectos: a) la definición económica de los "medios de subsistencia", "niveles de vida", "canasta de bienes y servicios" no agota la totalidad de las actividades relativas a la reproducción de los agentes sociales; b) dicha definición es un 'dato' social que varía según país, momento histórico y clase social, lo que la constituye también en un 'dato' para los miembros de cada clase y de las otras clases, es decir, en una probabilidad objetiva (valga la redundancia) que, de alguna manera, se hará presente como expectativa interiorizada por los miembros de las distintas clases y los motivará de distinta forma (al mantenimiento del poder social y sus privilegios, al ascenso social, a la subsistencia, etc.)

Si las distintas clases, capas o fracciones de ellas definen de distinta manera sus necesidades, tenderán a demandar satisfactores (bienes y servicios) que, a su vez, serán diferentes en cantidad y en calidad.

Se obtendría así la siguiente matriz de la demanda de bienes y servicios reproductivos:

\begin{tabular}{llllll}
\hline CLASE DE BIEN O & \multicolumn{4}{c}{ Sector social demandante } \\
\cline { 2 - 4 } & SERVICIO DEMANDADO & a & b & c ... & TOTAL
\end{tabular}

Alimentación

Vivienda

Educación

.........

TOTAL 
La sumatoria de cada hilera, es la demanda total de cada clase de bien o servicio. La sumatoria de cada columna, es la demanda total de bienes y servicios que hace cada sector social, sus medios de vida necesarios, su nivel de vida. La sumatoria de todas las celdas de la matriz, es la demanda global de bienes y servicios reproductivos.

Como se ha señalado, las unidades familiares son responsables de que sus miembros obtengan los satisfactores que necesitan, con variaciones según los parses, la clase de bien o servicio demandado y el sector social de que se trate. Empero, ellas no producen directamente la totalidad de los satisfactores por lo que es necesario tener en cuenta otras unidades intervinientes en la reproducción de los agentes sociales que constituyen lo que podria llamarse, la oferta de bienes y servicios reproductivos.

La organización social de la reproducción de los agentes sociales aparece, así, como un asunto clave para profundizar en el tejido conectivo existente entre las unidades constitutivas de la sociedad. renunciando a la "ficción de que existen microsociedades completas (o casi completas) dentro "del sistema global y a la postulación implícita de que el tejido societal "resulta de una sumatoria de unidades diferenciadas pero clasificables en "tipos" (Bartolomé, 1980) y para explorar algunas implicaciones ideológicas y políticas.

En efecto, en sociedades de clases, la demanda total de los distintos sectores sociales por cada clase de bien o servicio (alimentación, educación, salud, vestuario, vivienda, etc.) se atiende según distintos circuitos de satisfacción de las necesidades que incluyen una diversidad de unidades, agentes, prbcesos, prácticas, flujos e intercambios, que configuran sistemas más o menos lábiles y que no implican, necesariamente, modificaciones internas a las unidades que participan en ellos. En este sentido, son procesos de articulación social y, en cuanto tales, entre las unidades en ellos involucradas pueden darse relaciones conectivas verticales u horizontales; de articulación adaptativa, integrativa o de contradicción dialéctica; mediante mecanismos formales o informales. Cada proceso de articulación se caracteriza por una estructura y dinámica propias (Bartolomé, 1980).

La consideración conjunta e interrelacionada de las dimensiones política, organizativa, administrativa y técnica de la operación de los distintos circuitos, pone de manifiesto de qué manera la organización de la reproducción de los agentes sociales es, al mismo tiempo, un mecanismo de articulación entre las clases sociales.

La dimensión política es aquélla que apunta, directa o indirectamente, a orientar de manera juridicamente obligatoria para toda la sociedad, los distintos aspectos de la vida social. En ella se deciden las caracteristicas de la producción y las reglas de distribución del bien o servicio en cuestión (qué, a quiénes, cuánto, cómo y dónde). Así, por ejemplo, en un país de la región se adoptó la decisión política de desarrollar la educación básica y durante cincuenta años fue cerrada la universidad, con lo que, los sectores sociales altos, debieron satisfacer sus necesidades educativas en el extranjero. Por otro lado, las decisiones políticas pueden encontrar diversás dificultades al ser llevadas a la práctica. Así, por ejemplo, la adopción de una enseñanza básica común, gratuita y obligatoria a fin de lograr la homogeneidad míni- 
ma de los ciudadanos se enfrenta, en las zonas rurales, con la marcada heterogeneidad estructural y cultural, lo cual hace difícil su carácter de enseñanza común; se agrega a esto el hecho de que el trabajo de los menores es necesario, con lo que pierde sus características de gratuita, dada la significación de su trabajo para la economía familiar, y de obligatoria, dado que debe ceder su prioridad a otras urgencias y que su inobservancia es difícilmente sancionable.

La dimensión organizativa está constituida por el conjunto de posiciones en que se disponen los individuos como sujetos activos o pasivos en la ejecución, prestación o recepción de las acciones políticas, administrativas o técnicas. En la educación formal oficial, por ejemplo, se produce la forma de una pirámide (que frecuentemente incluye otras pirámides menores para la enseñanza básica, media, técnica) cuya cúspide está conformada por funcionarios de extracción política y con una rotatividad relativamente alta, cuyo tronco está compuesto por técnicos, supervisores, docentes y otros funcionarios y cuya ancha base está compuesta por el público (el alumnado y sus padres). Los flujos de comunicaciones y decisiones son predominantemente descendentes previéndose, a veces, la participación de los padres (limitada a colaboraciones económicas o a informaciones sobre el desempeño de sus hijos) y nunca, la del alumnado. Cuando los padres financian la educación de sus hijos, se presentan casos con distintas modalidades organizativas.

La dimensión administrativa está constituida por el conjunto de acciones tendientes a obtener, conservar, asignar y utilizar los medios para la ejecución de las políticas, el funcionamiento de la organización y sus prestaciones. Mediante ella se ponen diariamente en operación los aspectos políticos, organizativos y técnicos. Siguiendo con el ejemplo del sistema educativo" formal, la centralización de la administración en las capitales comienza por dar un sesgo urbano a todo el sistema (concentración de medios, redacción de libros de texto, proximidad a los problemas y a las fuentes de decisión). Aun dentro del nivel básico del sistema educativo formal oficial son patentes las diferencias entre las características de los circuitos destinados a las clases medias urbanas y a las zonas rurales empobrecidas: locales, medios didácticos, escuelas con todos los grados o con sólo algunos grados, docente a cargo de un solo grado o de varios, etc.

La dimensión tećnica está conformada por el personal que presta el servicio específico, su formación, sus prácticas, así como por la tecnología del equipamiento. En general, se ha comprobado que en las zonas rurales de varios países de la región hay una mayor proporción de docentes jóvenes, sin experiencia profesional u obligados a iniciarla en dichas zonas, que carecen de la capacitación necesaria para enseñar en las condiciones en que deben hacerlo y hasta de los métodos para la enseñanza de la lecto-escritura. Por otra parte, su formación enciclopedista, generalista, abstracta y verbal, se manifiesta en la forma en que ellos mismos enseñan. Por último, el niño rural que llega a la escuela proviene de unidades familiares que tienen ciertas condiciones de vida, cierto patrimonio cultural y están situadas en una ubicación social que implica una gran distancia respecto a los bienes y equipamientos culturales y a los centros de producción y transmisión de cultura. 
Las distintas prácticas que tienen lugar en el transcurso del proceso pedagógico incorporan las determinaciones propias de todas las dimensiones precedentemente consideradas. Por ello no es de extrañar, insistiendo con ejemplos educativos, el bajo rendimiento del sistema educativo formal, en particular en las áreas rurales, en las que no alcanza a cubrir a toda la población en edad escolar y en las que se registran altas tasas de repetición, deserción, abandono antes de aprobar el tercer curso y bajas tasas de promoción. El número de alumnos en edad escolar que son matriculados excede en mucho el número de alumnos que aprueba cursos, lo que indica, a la vez, una frustración por lo que se obtiene de la educación formal y una alta demanda de ellá. Cabe preguntarse, entonces, cuál es el papel que se adjudica a la educación en las estrategias familiares (por qué y para qué las familias demandan educación rural y cuál es el papel que juegan las estrategias familiares en las posibilidades educativas de sus miembros (de qué manera las unidades familiares condicionan la participación de sus miembros en el sistema educativo formal). Porque lo que parece estar fuera de duda es que, a pesar de que participan de un circuito de satisfacción de necesidades de baja calidad y del que obtiene también bajos rendimientos, las unidades familiares continúan demandando crecientemente ese satisfactor y, con ello, legitimando no sólo el circuito en el que participan sino, al mismo tiempo, los otros circuitos coexistentes.

Las acciones sociales que tienen lugar en el interior de los circuitos de satisfacción de necesidades, aparentemente dispersas, ponen en operación a la estructura social a través de las distintas prácticas que realizan los agentes sociales que pertenecen a distintas clases sociales y que ocupan diferentes posiciones en dichos circuitos, mediante las que se realizan los flujos e intercambios de bienes y servicios de consumo (instrumentales y simbólicos), de personal, de mensajes, entre las distintas unidades (hogares, sindicatos, empresas, iglesias, escuelas, mercados, etc.). (Berlinck, 1969).

Así, dada una esfera cualquiera de actividad y cualquier circuito de satisfacción de necesidades, las opciones de comportamiento de los agentes tienen una relativa regularidad y predictibilidad. Algunas de esas regularidades se vuelven tan visibles y estables que son susceptibles de ser formuladas como reglas de conducta que indican cómo comportąrse, con quién, dónde, es decir, el lugar que corresponde a cada una de las categorías de agentes actuantes en la sociedad (Bartolomé, 1980; Oliveira, 1976; Berlinck, 1969).

Las acciones e interacciones sociales son estructuradas y significantes para los agentes sociales y su aprendizaje 'natural' se produce, entre otros ámbitos, a través de los comportamientos que, como se señala precedentemente, se inducen a partir de la participación en las distintas posiciones que componen los diferentes circuitos de satisfacción de las necesidades.

Dadas ciertas condiciones (distancia social o geográfica, escasa visibilidad social, atomización de las unidades familiares, ideologías que enfatizan el consenso o justifican la desigualdad, etc.), hay distintas probabilidades de que se considere legitimo el circuito de satisfacción de necesidades en el que se participa, se ignore la existencia de otros circuitos o se legitime la existencia de circuitos diferenciales a través de los cuales se realiza la repro- 
ducción de los agentes sociales y, a través de ellos, la reproducción de las relaciones entre las clases sociales.

Si se consideran los circuitos de satisfacción de necesidades en su doble carácter de sistemas típicamente diferentes que configuran y articulan relaciones sociales, por un lado y, por el otro, de situaciones en las que los agentes sociales realizan aprendizajes acerca del lugar que ocupan en la sociedad $y$ de los comportamientos 'adecuados' a ese lugar (quien tiene y quien no tiene; quien sabe y quien ignora; quien manda y quien obedece; etc.) (Berger y Luckmann, 1972), dichos circuitos pueden ser tratados como "modelos socio-organizativos".

Quizás en los momentos en que se cuestiona la legitimidad de los circuitos de satisfacción de necesidades que existen y se proponen otros nuevos, es cuando se pone más claramente de manifiesto su carácter de modelos socio-organizativos, los que pueden analizarse en distintos aspectos, como por ejemplo:

a) Dónde se origina, propone o impulsa el cuestionamiento o la propuesta (el gobierno, algún partido político, alguna asociación intermedia, individuos aislados) y en conexión con qué clase o sector social;

b) En benefício o en perjucio de qué clases o sectores se produce el cuestionamiento o la propuesta y si contempla alguna modificación de las reglas de distribución del producto social;

c) Se limita a algún circuito de satisfacción de necesidades o apunta al cambio global de la organización social de la reproducción de los agentes sociales;

d) Se demanda una nueva clase de bien o servicio o el mejoramiento de la cantidad o calidad de la prestación de un bien o servicio al que ya se tenía acceso;

e) Se circunscribe a la dimensión política, administrativa, organizativa o técnica de alguno de los circuitos o se refiere a todas ellas en conjunto;

f) Se cuestiona el modo de generar la autoridad y las decisiones;

g) El modo de participación que se reclama en alguno de los aspectos del circuito de satisfacción de necesidades. (Borsotti, 1972; Borsotti, 1973).

Aceptando el riesgo de decir cosas obvias, si algo demuestra lo expuesto hasta aquí es que, aun reducida a los bienes y servicios consumidos por las unidades familiares o los individuos, la organización social de la reproducción de los agentes sociales es un fenómeno complejo. El haber centrado la atención en ella no implica desconocer la importancia de otros aspectos de la realidad social (la acumulación del capital, la formación de valor, las formas de dominación, etc.), pero sus relaciones recíprocas deberán ser dilucidadas.

El marco interpretativo que se ha propuesto es necesariamente abstracto y general y es notoria la necesidad de elaborar categorías más concretas y precisas para dar cuenta del problema. El objetivo de esta sección se habria cumplido si consiguiera llamar la atención sobre fenómenos particularmente descuidados. 


\section{LAS UNIDADES FAMILIARES}

Según lo expuesto en la sección anterior, en relación con la organización social de la reproducción de los agnetes sociales, las unidades familiares pueden considerarse por lo menos, desde dos puntos de vista:

a) Como unidades de decisión. Se trata de determinar las características del proceso de toma de decisiones acerca de las caracteristicas que tendrá la reproducción de todos o alguno de los agentes pertenecientes a la unidad familiar (quiénes toman las decisiones, cómo, respecto de qué miembro o miembros, etc.). Si efectivamente se determina que las unidades familiares se constituyen como unidades de decisión, se plantean serios problemas teóricos respecto de todos aquellos enfoques que dan por supuesto que el individuo no sólo es el consumidor final sino que es, al mismo tiempo, quien decide por si y ante si, qué consumir y cuánto (Muellbauer, 1974; Nerlove, 1974). Por cierto, hay que evitar el riesgo de suponer la existencia de decisiones explicitas y de procesos formales de decisión.

b) Como unidades de recursos mediante los cuales ejercen su responsabilidad en la reproducción de los agentes sociales. Sólo en el caso de unidades familiares aisladas puede pensarse que coincidan la unidad de decisión y la unidad de la que se obtienen todos los recursos reproductivos. En los casos restantes se recurre a alguna combinación de rédes de parentesco, redes de vecindario (Adler de Lomnitz, 1975), a la comunidad, a otras unidades sociales. Los indicadores para detectar las unidades a las que se recurre pueden ser varios: a quién se solicita un préstamo en dinero, información, influencia; a quién se recurre para el cuidado de los niños; a quién se invita a las fiestas y ceremonias, etc.

Pero en qué medida las unidades familiares son unidades de decisión y en qué medida obtienen recursos dentro de la misma unidad familiar o de otro tipo de configuraciones sociales, deja pendiente las preguntas centrales:

- ¿Qué es el fenómeno social que se denomina familia?

- ¿cuáles son sus variantes e invariantes?

- ¿qué papel ha tenido y tiene en distintas sociedades o en las distintas situaciones de clase de una misma sociedad, en relación con la organización social de la reproducción de los agentes sociales?

Esas preguntas no tienen respuesta por medio de la definición de las funciones de la familia, entre las que se ha incluido una diversidad de aspectos: la reproducción; la socialización; la gratificación sexual; la cooperación económica; la división interna del trabajo; la protección; la recreación; la distribución de status; etc. (Sussman, 1974). El asunto es que, saber cuáles son las funciones de algo no implica conocer en qué consiste ese algo.

La búsqueda de una definición genérica de las unidades familiares es, sin duda, una empresa difícil. Empero, hay algunas dimensiones sobre las que 
parece haber acuerdo básico cuando se trata de responder a las preguntas antes formuladas (el parentesco, el matrimonio, el hogar doméstico, la unidad de residencia, el grupo, etc.). La aproximación al fenómeno, por lo tanto, no puede producirse sin determinar cuál es la o las dimensiones que se aplican a la realidad en estudio, o si no puede ser aplicada ninguna. Eso dependerá, entre otras cosas del aspecto de la realidad que se quiere conocer . Conviene, entonces, analizar las dimensiones antes mencionadas para ver en qué medida ellas permiten delimitar un referente empirico que pueda ser considerado como unidad de decisión y de recursos en relación con la reproducción de los agentes sociales.

\section{Parentesco y matrimonio}

Como otros mamarios, la especie humana tiene dos sexos. La diferencia está en que el hombre presenta cursos alternativos en la manera de formar sus grupos, la herencia, los arreglos de pareja, etc. Estudiar el parentesco es estudiar lo que se hace y por qué y las consecuencias de adoptar una alternativa en vez de la otra. El parentesco es la relación entre personas según la consanguineidad real, putativa o ficticia. En definitiva, un consanguíneo es alguien a quien la sociedad define como tal, por lo que algunos distinguen entre pater y genitor y mater y genitrix (Fox, 1967). Por su parte, las normas de acoplamiento designan los matrimonios posibles (Meillassoux, 1979). El matrimonio es una alianza, pero marido y mujer no son parientes, aunque esa alianza pueda estar también regulada por el parentesco y defina parentescos. Las normas de filiación indican las relaciones de dependencia de un individuo frente a las generaciones anteriores (Meillassoux, 1979).

El hecho de que en el matrimonio el varón y la mujer aparezcan como individuos o como representantes de grupos, no es indiferente. Es más, las normas de acoplamiento, entre las que se cuenta al tabú del incesto, fijan los límites de las uniones (alianzas) matrimoniales permitidas o prohibidas, raramente prescritas, lo que enfatiza el carácter de alianza y no de parentesco de la unión matrimonial. Cuando se hace presente el imperativo de la alianza entre no parientes establecida por unión matrimonial, aparece reforzado por una serie de mitos que muestran las consecuencias nefastas de las uniones entre parientes, pocas de las cuales son científicamente demostrables: monstruos, defectos en.la sangre, idiotismo, etc.

En las sociedades en que la elección del cónyuge es aparentemente libre, es necesario determinar las reglas de endogamia y exogamia que regulan ese mercado (edụcación, status socioeconómico, edad, etc.).

El vínculo de parentesco y la alianza matrimonial definen una serie de derechos y obligaciones recíprocas (legales y socialmente vigentes), que, por cierto, no se extienden a todos los parientes que están vivos. Por eso es necesario definir los límites de las redes de parentesco: entre qué parientes se definen derechos y obligaciones recíprocas; entre cuáles de esos parientes se ponen efectivamente en práctica en la vida real; cuál es el contenido de esos derechos y obligaciones. En este sentido, se ha señalado la diferencia que existe, en sociedades transicionales-metropolitanas, entre las estructuras de 
parentesco de la élite, la clase media y la clase baja (Berlinck, 1969). Asimismo, se ha hecho notar que la importancia de una familia extensa no es su composición, sino más bien las relaciones y obligaciones recíprocas que implica, citándose el caso del compadrazgo y entre quiénes se busca a los compadres en las distintas clases sociales. En este mismo estudio se sostenía que la modernización puede afectar a los individuos de las redes de parentesco, sin necesariamente destruir a las redes mismas (Carlos y Sellers, 1972).

Esta dimensión de las redes de parentesco, permite definir un referente empírico que parece ser ineludible en cualquier estudio de las unidades familiares y su papel en la organización de la reproducción de los agentes sociales, tanto como unidades de decisión cuanto como unidades de recursos: cómo se constituyen; cómo se mantienen; y los derechos y obligaciones efectivamente vigentes que constituyen y mantienen las redes de parentesco.

\section{Unión matrimonial, relaciones sexuales y reproducción}

Hay una serie de distinciones que, aunque conocidas, es necesario tener presentes: a) las relaciones eróticas no son equivalentes a las relaciones sexuales; b) no toda relación sexual culmina en procreación y se ha registrado un proceso histórico por el cual se ha producido una separación entre el placer sexual y la unión sexual reproductiva; c) no toda relación sexual que culmina en procreación da origen a una unión matrimonial o se realiza en el marco de ella.

¿Cuándo una relación sexual heterosexual puede ser considerada unión matrimonial? Se ha propuesto que el matrimonio es una relación estable en la que se permite socialmente a un hombre y a una mujer tener hijos sin pérdida de su reputación en la comunidad (Johnson, 1965). Hay aquí dos componentes básicos: la aceptación social y la estabilidad. Se ha hecho notar que la aceptación social se relaciona con el control del amor y que son necesarias muchas energías y recursos para canalizar a los enamorados hacia una conducta de rol adecuada respecto del amor, por lo que éste debe ser controlado antes que aparezca. Sólo si la línea de parentesco no es importante en una sociedad como un todo, la elección será enteramente libre aunque, en sociedades estratificadas, la importancia de mantener líneas de parentesco intactas y aceptables será mayor en los estratos altos, que tienen más interés en el mantenimiento de la estructura social y están más motivados en el control del cortejo y el matrimonio (Goode, 1959). La aceptación social no se confunde con la formalidad de la unión. Puede convenirse que toda unión formal es socialmente aceptada, pero la aceptación social de una unión no formalizada sólo puede determinarse en el exterior de dicha unión.

En lo que hace a la estabilidad de la unión, se plantea el problema de los umbrales, es decir, de cuánto debe durar una unión para que se la considere como unión matrimonial.

Por otro lado, en el caso de las uniones matrimoniales que se disuelven y en que cada uno de los miembros vuelve a entrar en una nueva unión matri- 
monial, se plantea el problema de la adjudicación de los hijos tenidos en las uniones sucesivas.

En relación con las unidades familiares en cuanto unidades de decisión y de recursos en la organización social de la reproducción de los agentes sociales, esta dimensión también permite definir un referente empírico, el que parece tener particular importancia en aquellos sectores sociales en que son frecuentes las uniones inestables y sucesivas.

\section{La unidad doméstica}

El concepto de hogar o unidad doméstica es más amplio que el de compartir la obtención, elaboración y distribución de alimentos. Esto se refleja incluso en las propias definiciones censales: proveer sus necesidades alimenticias y otras esenciales para vivir, cocinar y comer en conjunto (Argentina) vivir juntos y compartir al menos una comida diaria (Barbados); hacer vida en común bajo un régimen familiar, compartir la vivienda y tomar juntos sus comidas (Costa Rica); vivir junots compartir, "common living arrangements", pueden compartir o no sus comidas (Ja maica); vivir bajo un régimen familiar o parecido (Pa raguay). (OEA, 1970). De estas definiciones queda en evidencia que el concepto de unidad doméstica no coincide con el de la unidad familiar definida según el parentesco, el matrimonio o la residencia (Sussman, 1974).

Una conocida antropóloga presentaba un caso que consideraba incluido dentro del concepto de unidad doméstica. Se trataba de una mujer sola, con varios hijos, a la cual un grupo de hombres había provisto de una cocina y le proporcionaban el dinero para la compra y elaboración de los alimentos. Este servicio era retribuido en alimentos. Es decir, por su trabajo al efectuar las compras de viveres y cocinar, la mujer obtenia su alimentación y la de sus hijos. (Cardoso, 1979). A menos que el concepto de hogar o unidad doméstica se restrinja a los aspectos puramente alimenticios, pareciera que en el caso presentado no hay una unidad doméstica, sino varias: la constituida por la mujer con sus hijos, cuya necesidad alimenticia se atendía con el servicio prestado por ella, quedando por determinar cómo resolvía la atención de los otros rubros; la o las unidades domésticas de los hombres que pagaban el servicio, ya que podían atender en conjunto o por separado a los otros rubros de sus necesidades.

El concepto de unidad doméstica muestra, así, sus propias ambigüedades y será necesario tener en cuenta entre quiénes se conforman un ingreso común, entre quiénes se opera el gasto de ese ingreso de acuerdo a un presupuesto en común, qué rubros se incluyen en ese presupuesto común, qué parte de sus ingresos se reservan para sus gastos personales los que participan en el ingreso y el gasto hogareño, etc.

Esta dimensión, aun reducida a sus aspectos puramente económicos (quiénes participan en la conformación del ingreso de la unidad familiar, entre quiénes se distribuye el consumo de bienes y servicios que se atiende con ese presupuesto), permite definir un referente empírico que parece ser ineludible para estudiar a las uniciades familiares en cuanto unidades de de- 
cision y de recursos en la organización social de la reproducción de los agentes sociales. Sin ella, difícilmente puedan comprenderse fenómenos tales como la adopción de decisiones económicas, la organización del trabajo doméstico, la participación en el mercado de trabajo, la distribución del ingreso que se realiza en el interior de las unidades familiares, etc.

\section{La residencia en común}

Las pautas de residencia indican el lugar donde vivirán las uniones matrimoniales. Las reglas básicas son la residencia patrilocal, matrilocal, bilocal, avuncular, neolocal. (Johson, 1965). La pauta de residencia puede referirse a todo el ciclo familiar sólo a algunas etapas de éste. Así, una pareja recién unida puede vivir con los padres de alguno de ellos hasta que puedan tener su propia vivienda, sin que exista ninguna pauta expresa acerca de qué deba hacerse, así no haya prescripciones sobre la patri o la matrilocalidad. En algunas culturas, las uniones no se concretan hasta que la pareja no puede construir su vivienda. Por su parte, las "uniones de visita", de las que informa la literatura proveniente del Caribe, consisten básicamente en el hecho de que los compañeros sexuales (en uniones relativamente estables que suelen tener hijos), no compaten la residencia.

El predominio de la residencia neolocal ha sido tomada como prueba de la existencia de una pauta de organización familiar en que predomina la familia nuclear aislada y ambos hechos han sido asociados a los procesos de urbanización e industrialización. Por un lado, se ha sostenido que no hay relación causal necesaria y suficiente, sea expresada en términos de interdependencia o consecuencia funcional necesaria, entre la pequeña familia nuclear y la urbanización y la industrialización (Greenfield, 1961-1962). Por otro lado, la residencia neolocal suele establecerse cerca de parientes con los que se intercambian frecuentemente bienes y servicios.

$\mathrm{El}$ análisis de las unidades de residencia según su estructura de parentesco y el tamaño y composición por sexo y edad, ha sido objeto de especial atención por parte de la demografía de la familia (Burch, 1974, 1976, 1979; García, Muñoz y de Oliveira, 1979. a, b y c.; Lira, 1976, a. y b.; Lopes, 1976; Pantelides, 1976; Siegel, 1976; Taueber, 1973, United Nations, 1973; Westoff, 1974). La estructura de los hogares censales según la relación de parentesco que tienen con el jefe las otras personas presentes, da como resultado una gran variedad de estructuras posibles, aunque se considere sólo la presencia del jefe, su cónyuge, hijos solteros, hijos casados, nietos, padres o suegros y otros parientes (Recchini de Lattes y Borsotti, 1980). El hecho de que alguna estructura en particular registre porcentajes muy altos en la distribución nacional, no implica que deba desconocerse la existencia de otras estructuras que estén asociadas a ciertas zonas de residencia, situaciones de clase, etapas del ciclo familiar, sexo o edad del jefe. Por otro lado, la composición por sexo y edad de las distintas estructuras de hogares censales y su asociación con otras variables, implica considerar a las unidades familiares como unidades de decisión o de recursos. (García, Muñoz y De Oliveira, 1979 , a, b. y c.) 
En todo caso, el solo hecho de convivir es un fuerte indicador de la existencia de algún tipo de relación, derechos y obligaciones entre quienes comparten la unidad de residencia y, en base a él, puede constituirse un referente empírico de importancia para comprender el papel de las unidades familiares en la organización social de la reproducción de los agentes sociales.

\section{El grupo social}

La familia suele ser considerada como un grupo de pertenencia pequeño, de interacción primaria, es decir, cara a cara. A pesar de su importancia, es menos frecuente que se incluya la connotación de comunidad, en cuanto sentimiento de pertenencia a un todo.

La caracterización de pequeño grupo, puede ser acertada por aquéllos que comparten la unidad de residencia y, en particular, para las unidades familiares nucleares mientras los hijos no se han ido de su familia de orientación o no han formado su familia de procreación, todo según las definiciones culturales vigentes. Sin embargo, un hijo migrante, que tiene su propia familia de procreación y que remesa dinero a sus padres, sigue perteneciendo a su familia de orientación definida según los derechos y obligaciones del parentesco, pero no sigue perteneciendo a ella, definida según las características del pequeño grupo de interacción primaria.

Los límites formales del grupo están definidos por el matrimonio y el parentesco. Los límites efectivos del grupo de pertenencia, están marcados por los derechos y obligaciones recíprocos, referidos a la unidad doméstica o a la unidad de residencia. Los límites efectivos del grupo de referencia no se definen por la pertenencia actual al grupo, sino por la vigencia efectiva de los derechos y obligaciones recíprocos de la unidad familiar con miembros ausentes, normalmente fundados en el parentesco.

Las necesidades del ser humano recién nacido; los intercambios inmediatos o diferidos que se registran en el marco del pequeño grupo en el que crece; los poderosos vínculos psicológicos de simbiosis, lealtad, rechazo, que se generan (García Badaracco, 1980) hacen que esta dimensión también aparezca como importantes para definir un centro de decisiones y un núcleo de recursos y, por tanto, para comprender el papel de las unidades familiares en la organización social de la reproducción de los agentes sociales.

\section{A manera de sintesis sobre el tema}

En este momento hay que retomar los interrogantes planteados al comienza de esta sección, acerca de qué es el fenómeno social que se denomina familia, sus variantes e invariantes, su papel en distintas sociedades o en las distintas situaciones de clase de una misma sociedad.

El referente empírico denotado por la expresión 'familia' puede tener una gran variación, pero eso es propio de cualquier otra unidad o institución social y no justifica aproximaciones epistemológicas de una indeterminación total. Como se ha hecho notar, una teoría del desarrollo familiar no surgirá de la acumulación de monografías (Lasch, 1975). Sólo si se toma conciencia 
de los problemas de método comparativo, la necesidad de seleccionar categorías adecuadas de análisis y se utilizan los controles pertinentes, se pueden empezar a entender los determinantes de la estructura familiar, sean ellos políticos, legales, demográficos, sociales o económicos (Berkner, 1973). Los estudios sobre grupos o localidades particulares que no reúnan esas condiciones, van acumulando conocimiento pero, como sucede con los 'casos' o 'ejemplos', sólo prueban algo si contradicen las hipótesis.

La multidimensionalidad de las unidades familiares es un rasgo que dichas unidades tienen en común con cualquier otra unidad social, ya que todas ellas son relaciones sociales (comunalizaciones o socializaciones, según Weber), que existen en la medida en que los partícipes en ellas orientan reciprocamente su acción por esa pertenencia. En las unidades familiares, los fundamentos de esa orientación recíproca pueden basarse en el parentesco, la unión matrimonial, la residencia en común, la conformación de un hogar o comunidad doméstica, los lazos resultantes del pequeño grupo.

El problema reside en la falta de univocidad con que se usan los términos, y algunas expresiones frecuentes, son decididamente incorrectas. En estudios basados en datos censales se lee a menudo que "un tanto por ciento de las familias son nucleares", cuando en realidad, lo que debiera decirse es que "un tanto por ciento de las unidades de vivienda están ocupadas por estructuras nucleares de parentesco", o que "un tanto por ciento de los hogares censales están formados por estructuras nucleares de parentesco".

La propuesta de dar preferencia a las unidades domésticas y descuidar las otras dimensiones de las unidades familiares no parece del todo adecuada. La unidad doméstica, en cuanto conformación común de un ingreso y un presupuesto para atender a las necesidades, presenta el riesgo de un cierto reduccionismo económico que puede arrastrar por la pendiente del cálculo del costo-beneficio, con toda una concepción del 'homo oeconomicus' subyacente y a descuidar otras dimensiones de la reproducción de los agentes sociales. Debe tenerse en cuenta que, para la gran mayoría de los casos, la motivación para la constitución de unidades domésticas y de unidades de residencia, puede basarse, en último término, en los derechos y obligaciones derivados del parentesco y del matrimonio.

En consecuencia, es recomendable que los estudios sobre el papel de las unidades familiares en la organización de la reproducción de los agentes sociales, dediquen la mayor atención posible a precisar cuál o cuáles de las dimensiones antes analizadas constituyen el objeto de la investigación. Esto permitirá disponer de un referente empírico delimitado conceptualmente, acumular conocimientos sobre las variantes e invariantes del fenómeno familiar, decidir cuál es la importancia relativa de las distintas dimensiones en relación con la organización social de la reproducción de los agentes sociales y, 'last but not least', evitar confusiones terminológicas.

\section{LAS ESTRATEGIAS FAMILIARES}

Hablar de estrategias familiares o de estrategias de las unidades familiares implica plantear varios supuestos referidos a las unidades, los recursos con que 
cuentan, el medio en que actuán, las acciones que emprenden, los objetivos que persiguen y una racionalidad estratégica. Es conveniente considerarlos por separado:

a) Como sucede con cualquier otra institución social, los comportamientos visibles consisten en conductas de los individuos (quién hace qué, cómo, cuándo, dónde, con qué instrumentos, con qué resultados, etc.). Por lo tanto, hablar de estrategias de las unidades familiares significa que se da por sentada la existencia de alguna forma de unidad de decisiones, de puntos de referencia, de fundamento para la orientación de las acciones. En suma, la existencia de una unidad a la que se imputa la estrategia, cuyos límites respecto de otras unidades similares, más o menos lábiles, deben ser determinados. Esos límites pueden establecerse poniendo en relación las dimensiones antes expuestas (parentesco, matrimonio, unidad doméstica, unidad de residencia, grupo) y la responsabilidad por la reproducción de los agentes sociales. La determinación de una unidad de imputación, no implica ningún supuesto acerca de la ausencia de conflictos o contradicciones en el interior de dicha unidad.

b) Los recursos con que cuentan las unidades familiares son diversos: número de personas, tiempo, ingresos, relaciones, trabajo, ocio, capacidad o incapacidad de postergar gratificaciones, horizonte temporal, bienes, otras unidades sociales similares o diferentes, etc. Los recursos, cuya diversa naturaleza queda de manifiesto en la incompleta enumeración precedente, varían según el país, la situación de clase y la zona de residencia de las unidades familiares. No puede plantearse el supuesto de que las unidades familiares tienen conciencia de todos sus recursos. Es más, es importante retener las diferencias entre recursos potenciales, accesibles y utilizados.

c) El medio en que actúan las unidades, cuyas estrategias sólo pueden ser comprendidas si se ponen en relación con otros componentes sociales (facilitadores, indiferentes u opositores) y, en particular, con los siguientes hechos: i) las fuentes accesibles de ocupación e ingresos para las distintas categorías de miembros de la unidad; ii) la definición social de la canasta de bienes y servicios a la que pueden acceder según su situación de clase; iii) los circuitos de satisfacción de las necesidades a los que tiene acceso; iv) los objetivos y motivaciones culturales (consumo inmediato, consumo diferido, ascenso social, individual o grupal, etc.) Como se ha hecho notar, puede encontrarse aquí una de las fuentes de la alienación o de la falsa conciencia de clase (GEA, 1980). No puede darse por supuesto que las probabilidades objetivas existentes en el medio hayan sido incorporadas como expectativas subjetivas por los miembros de las unidades.

d) Las acciones que emprenden la unidad o sus miembros, consistentes en los hechos más diversos, de las que no puede suponerse un mismo grado de conciencia en relación con los objetivos.

e) Los objetivos que se persiguen constituyen el punto central del tema de las estrategias familiares: cuáles son; cómo se determina cuáles son; cómo se imputan; en qué medida las acciones de los miembros son congruentes o incongruentes con los objetivos que se imputan a las unidades familiares. Algunos ejemplos aclararán algunos de los problemas que se presentan. Se 
sostiene que las distintas estructuras familiares hacen distintas presiones sobre los miembros individuales de la familia para que progresen en la sociedad (Sussman, 1974). Esta proposición puede leerse, al menos, de dos manenras: i) el objetivo de progresar es común a todas las estructuras familiares y las presiones que ejercen sobre sus miembros varian según dichas estructuras; ii) algunas estructuras familiares tienen como objetivo progresar en la sociedad, para lo cual ejercen distintas presiones sobre sus miembros. También se sostiene que las estrategias de supervivencia están encaminadas a asegurar la reproducción material y biológica del grupo social (PISPAL, citado en Argüello, 1980). Si se trata de la reproducción de la situación de clase de la unidad familiar, esto parece haberse demostrado para los estratos populares metropolitanos (García, Muñoz y De Oliveira, 1980). La reproducción de la situación de clase y del tipo de unidad familiar no se ha demostrado para otros sectores (Archetti, 1957; Marshall, Wolfe, en Borsotti, 1978, págs. 44 y 45).

f) La racionalidad implicita en toda estrategia supone que las acciones que se emprenden, dados ios recursos y el medio, tienden a lograr los objetivos de las unidades.

Como puede verse, los supuestos implícitos son fuertes, pero es necesario enfrentarlos si se quiere dejar de lado una visión de las unidades familiares según la cual éstas actúan de manera aleatoria y aislada, en la coyuntura y en el nivel denominado 'micro' y alcanzar un nivel de análisis en el que, a través de la determinación del papel que corresponde a las unidades familiares en la organización social de la reproducción de los agentes sociales, se acceda a la comprensión de los mecanismos de articulación y de reproducción de la sociedad.

Antes de proseguir, parece conveniente ilustrar las dificultades con algunos ejemplos. Con respecto a las redes de parentesco, se ha hecho notar que, para mantenerlas hace falta tiempo y dinero y que ambos recursos son escasos en la clase baja. En cambio, la estructura extensa de parentesco en las clases altas y medias altas, es un mecanismo adaptativo viable y se mantiene en el sistema. El control familiar de las decisiones clave siempre ha sido un elemento importante para las oligarquias tradicionales. Para obtener la información necesaria, hay que ocupar las posiciones del sistema con gente confiable y nadie más confiable que un pariente (Berlinck, 1969). Se ha sostenido que, ante todo, la oligarquía es una red de familias que controlan la riqueza (Carlos y Sellers, 1972). Cabe preguntarse si las redes de parentesco de la oligarquía operan de la manera descrita en cualquier tiempo y lugar, y, en todo caso, cuáles son las bases sobre las que se considera confiable a la gente. Por otro lado, dada la escasez de tiempo y dinero de las redes de parentesco que se dice que tienen las clases bajas, surgen las preguntas acerca de si efectivamente esas redes han desaparecido $y$, en ese caso, cuáles son las redes (si las hay) que las reemplazan.

Con respecto al número de hijos, se ha propuesto una interpretación de su racionalidad según se trate de familias burguesas, rentistas, empresarias, gerenciales, proletarias, pequeño burguesas, marginalizadas (Singer, s. d, b). Con ello se responde, de alguna manera, a la pregunta sobre a partir de 
qué número una familia es grande o chica y grande o chica para qué (Burch, 1974). En lo que respecta a las familias grandes de los sectores de subsistencia urbano y rural, se informa que ellas han sido objeto de las siguientes interpretaciones: i) son una elecciớn racional pero no se aclara respecto de qué objetivo y en qué circunstancias; ii) son un requerimiento y no una alternativa, con lo que, dentro de un fatalismo social, se deja sin establecer quiénes formulan el requerimiento y a quiénes; iii) son beneficiosas social y económicamente, pero no se explicita en qué consiste el beneficio, ni quiénes serían los beneficiados (Giffin, 1980).

Es conveniente, también, recordar los distintos estudios sobre las unidades familiares rurales, en especial las del campesinado, y la diversidad de hechos registrados: producción para auto-consumo y para el mercado; trabajo predial y extrapredial; migraciones temporales o permanentes; trabajo de las mujeres y de los menores; número de hijos; educación, etc. (Gómez, 1980); González, 1980; Molina Barrios, 1980, Palau, 1980).

Teniendo presente, entonces, la diversidad de acciones y de dimensiones que se encuentran en cada unidad familiar, hay que retomar las preguntas anteriormente planteadas; en qué consisten las estrategias familiares y cómo abordar su estudio.

La estrategia enseña el uso de los encuentros aislados y las maneras de combinarlos unos con otros para alcanzar el objetivo de la guerra (Clausewitz, 1976).

"Las causas que condicionan el uso del encuentro en la estrategia pueden ser divididas convenientemente en elementos de diferentes clases. Estos elementos son múltiples y están relacionados íntimamente unos con otros en cada operación aislada de la guerra, por lo que no puede tratarse la estrategia conforme a los elementos por separado. En tal caso, nos perderíamos en un análisis más desarticulado y, como en una pesadilla, buscaríamos siempre en vano levantar un arco que relacionara esta base de abstracciones con los hechos pertenecientes al mundo real". (Clausewitz, 1976, el subrayado es nuestro). En esta línea de pensamiento, se ha notado que "si el objetivo es contentarse "con un catálogo de conductas orientadas a la obtención de ingreso, no será "posible dar cuenta de dimensiones políticas u organizativas bajo las cuales tenderá a manifestarse la necesidad de satisfacer demandas básicas" (Palau, 1980). Así como un general desconoce, normalmente, la estrategia del adversario y trata de inferirla a traves de las distintas acciones que éste emprende sin dejarse engañar por ellas, del mismo modo se trata de inferir la racionalidad, la lógica, subyacente en el cúmulo de comportamientos de las unidades familiares y de sus miembros. EL objetivo, es claro, no puede consistir en contentarse con un catálogo, ni puede esperarse a que éste se complete, sino en una lectura del dato que vaya más allá de su referente empírico inmediato para comprender su lógica dentro de la situación en que se inserta.

Esto implica partir de la conducta como signo y rigurosamente observada, tomar los comportamientos como medios, como manifestaciones, indicios o pistas.

Al hablar de conductas, se está haciendo referencia a un campo que no se. 
reduce a las verbalizaciones, sino a todo tipo de emergentes conductuales. Toda conducta, cualquiera sea su grado de regularidad, es una manifestación más o menos directa, de ciertos principios a partir de los cuales se organiza, e implica que quienes la realizan le dan significado o sentido, la valoran, desde el ángulo particular de su propia inserción en un proceso social. Esto no significa que los individuos que las ponen en práctica sean conscientes de los principios de organización que están utilizando. En la relación entre el signo y lo significado, hay distintas formas de signos y de significaciones y distintas maneras de articularlos. Hay hechos que implican afirmaciones contradictorias: el minifundista que migra estacionalmente para obtener un ingreso monetario afirma que, para seguir siendo productor 'independiente' debe ser a la vez un asalariado; el emigrante que remesa dinero a su familia de origen, afirma la continuidad de la unidad familiar mediante su desarticulación; el obrero que manda a sus hijos a la universidad, afirma su capacidad de hacerlo y su deseo de que sus hijos no reproduzcan su propia situación, etc.

Se trata, en suma, de describir conductas y, por ensayo y error, ir proponiendo hipótesis acerca de los principios que organizan esas conductas aparentemente dispersas, sus propias formas de racionalidad, su lógica, para luego someterlas a prueba y explicarlas, recordando que "en un mecanismo "psico-cultural, son tan importantes los elementos presentes como los ausentes" y que "los diferentes elementos se configuran como una 'Gestalt': cada uno "adquiere su significado por su articulación con los otros y en el todo" (Aduriz, 1972). En relación con esa hipótesis se procede a la organización y a la lectura de los datos. Si se considera bien, no hay en esta propuesta nada distinto de lo que sucede cuando se estudia cualquier otra organización social y se vuelve a encontrar aquí la carencia de una metodología y una técnica sociológica sistematizada. Hay métodos y técnicas para estudiar distintos aspectos parciales (el sistema de posiciones, los grupos formales e informales, el flujo de comunicaciones, formas de obtención de los recursos, modos de colocación del producto, etc.), pero dichos métodos y técnicas no están sistematizados para relacionar esos aspectos parciales con la organización como unidad.

En síntesis, las unidades familiares, según su situación de clase y el medio en que están insertas, movilizan y organizan sus recursos para el logro de ciertos objetivos referidos a la unidad o a sus miembros, cualquiera que sea el grado de conciencia que éstos tengan acerca de esa organización y esos objetivos. Las estrategias familiares son la reconstitución de la lógica subyacente en todos esos hechos.

La noción de estrategias familiares es el nexo entre la organización social de la reproducción de los agentes sociales (con sus distintos circuitos de satisfacción de necesidades) y las unidades familiares responsables de dicha reproducción.

\section{IV: IMPLICACIONES}

En las tres secciones precedentes se han expuesto un marco aproximativo para interpretar la organización social de la reproducción.de los agentes sociales, 
un análisis de las distintas dimensiones de las unidades familiares en cuanto responsables de la reproducción de sus miembros en el ciclo cotidiano y generacional y, una forma de concebir y determinar las estrategias implícitas en la diversidad de acciones que emprenden dichas unidades o sus miembros. Cada una de esas secciones contiene, de alguna manera, sus conclusiones por lo que ahora, más que volver sobre lo expuesto, se harán notar algunas implicaciones.

1. Debe evitarse dar por supuesto que existe una relación unívoca entre los circuitos de satisfacción de necesidades, la situación de clase a la que pertenecen las unidades familiares y sus estrategias. Habría que determinar de qué manera esas relaciones varían según los paises y los distintos tipos de bienes y servicios y se registran distintos grados de desigualdad en los circuitos de satisfacción de necesidades educativas, de salud, de vivienda, etc.

2. Dada la diversidad de dimensiones de las unidades familiares y de tipos de bienes, servicios y actividades involucradas en la organización social de la reproducción de los agentes sociales, es ineludible en los estudios que se emprendan, la cooperación de varias disciplinas. Para que esta cooperación no se convierta en una sumatoria de capítulos estancos, como suele suceder hasta el presente, es necesario avanzar en la elaboración de marcos interpretativos interdisciplinarios. Puede comenzarse haciendo una lectura de estudios específicos ya realizados, que tenga en cuenta, a la vez, los modelos socio-organizativos implicitos en los circuitos de satisfacción de necesidades y la lógica de las estrategias familiares.

3. Desde un punto de vista descriptivo del papel de las estrategias de las unidades familiares en la organización social de la reproducción de los agentes sociales, interesan predominantemente estudios empíricos centrados en la forma en que las unidades familiares de las distintas clases sociales se insertan en los circuitos de satisfacción de necesidades de los distintos tipos de bienes (educación o vivienda o salud o alimentación, etc.), lo que implica:

a) Definir las unidades pertinentes, teniendo en cuenta sus diversas condiciones institucionales (por ejemplo, en el ámbito rural, la escuela es la concreción del sistema educativo formal nacional);

b) Establecer cuáles son los roles mediante los cuales se realizan las conexiones entre las unidades familiares y las otras unidades de los circuitos de satisfacción de necesidades, su relación con el sistema local y nacional de posiciones;

c) Analizar cuáles son las prácticas de los distintos participantes en el sistema de posiciones;

d) Determinar cuáles son los recursos que se utilizan y las características de su circulación entre las distintas unidades;

e) Establecer las orientaciones generales que dan origen a las actitudes y condicionan las estrategias de los actores individuales y colectivos y que estructuran el contexto cultural de la acción, en particular, las formas en que los circuitos de satisfacción de necesidades son legitimados o cuestionados;

f) Considerar la dimensión temporal en la que se inscriben las relaciones entre las unidades articuladas, las modificaciones que se hubieran registra- 
do en esas relaciones y las situaciones sociales específicas que estimulan o frenan los procesos de articulación. (Bartolomé, 1980).

4. A la demografía le cabe un papel fundamental, no sólo desde el punto de vistà descriptivo y cuantitativo (tamaño de los sectores involucrados en los distintos circuitos, según edad, sexo, características económicas, etc., de la población; características de los hogares censales, etc.), sino también en la determinación de los condicionamientos recíprocos entre esos circuitos y la fecundidad, mortalidad, morbilidad, nupcialidad, etc., de los sectores correspondientes de la población. (Corsa and Oakley, 1972).

5. Las políticas sociales y la planificación social, hasta el presente, se han orientado predominantemente por tipos de bienes y servicios (sectorialización por vivienda, salud, educación, etc.), y no han tenido suficientemente en cuenta la existencia de distintos circuitos de satisfacción de necesidades y sus implicaciones políticas, organizativas, administrativas, técnicas e ideológicas. Mientras las políticas sociales y la planificación social no incorporen adecuadamente la coexistencia de circuitos diferenciales, seguirán haciendo el supuesto implícito de que en el interior de cada circuito o entre circuitos se mantendrán las mismas formas socio-organizativas, con las consecuencias ideológicas y políticas señaladas.

\section{BIBLIOGRAFIA}

ADLER DE LOMNITZ, Larissa. ¿Cómo sobreviven los marginados?, México, Siglo XXI, 1975 .

ADURIZ, Joaquín. Así viven y asi nacen. En: REVISTA LATINOAMERICANA DE ESTUDIOS URBANO REGIONALES, EURE, Santiago , Centro de Desarrollo Urbano y Regional, v. II, n. 5, julio 1972, pp. 107-133.

ARCHETTI, Eduardo P. y Kristi Anne STOLEN. Explotación familiar y acumulación de capital en el campo argentino. Buenos Aires. Siglo XXI, 1975.

ARGENTINA, República. Ministerio de Economía. Secretaria de Estado de Programación y Coordinación económica. Instituto Nacional de Estadística y Censos. Encuesta de presupuestos familiares, 1969/1970. Buenos Aires, Instituto Nacional de Estadística y Censos, 1970. Serie investigaciones demográficas, 7.

ARGÜELLO, Omar. Delimitación del concepto "Estrategias de supervivencia" y sus vínculos con la investigación socio-demográfica. Comunicación al Taller sobre Estrategias de supervivencia. Buenos Aires, 13 y 14 de marzo de 1980. Borrador para discusión, mecanografiado.

BARTOLOME, Leopoldo J. Sobre el concepto de articulación social. En DESARROLLO ECONOMICO, v. 20, n. 78, julio-septiembre, 1980, pp. 275-286.

BEAUFRE, General. Estrategia de la acción. Buenos Aires, Pleamar, 1973.

BERGER, Peter L. y Thomas LUCKMANN. La construcción social de la realidad. Buenos Aires, Amorrortu, 1972, 2da. ed.

BERKNER, Lutz K. Recent research on the history of the family in Western Europe. En: JOURNAL OF MARRIAGE AND THE FAMILY, v. 35, n. 3, August, 1973, p. $395-405$.

BERLINCK, Manoel Tosta. The structure of the brazilian family in the city of Sao Paulo. En: LATIN AMERICAN STUDIES PROGRAM. Dissertation Series, s. 1. Cornell University, February, 1969 , n. 12.

BORSOTTI, Carlos A. Demandas, modelos organizativos y proyectos sociales. Santiago ELASFLACSO, noviembre de 1972, miemo. 
BORSOTTI, Carlos A. Documento para la discusión del marco teórico de la investigación "Estrategias de supervivencia de las unidades familiares de los sectores populares urbanos. Santiago, ELAS-FLACSO, mayo de 1973, mimeo.

BORSOTTI, Carlos A. Estudio preliminar sobre las relaciones entre educación y desarrollo en la sociedad rural latinoamericana. Buenos Aires, Proyecto "Desarrollo y educación en América Latina y el Caribe", 1980. mimeo.

BORSOTTI, Carlos A. Notas sobre la familia como unidad socioeconómica. Santiago, CEPAL, 1978, Cuadernos n. 22.

BORSOTTI, Carlos A. Estilos de desarrollo, medio ambiente y estrategias familiares. Santiago, Proyecto "Estilos de desarrollo y medio ambiente en América Latina", 1979.

BURCH, Thomas K. Consideraciones sobre el análisis de la estructura del hogar y de la familia. En: BURCH, Thomas K., Luis Felipe LIRA y Valdecir LOPES: "LA FAMILIA COMO UNIDAD DE ESTUDIO DEMOGRAFICO”. San José, CELADE, 1976, pp. 123140.

BURCH, Thomas, K. Estructura comparativa de la familia: un acercamiento demográfico. Santiago, CELADE, 1974, mimeo.

BURCH, Thomas K. Household and family demography: a bibliographic essay. En: POPULATION INDEX, April, 1979, v. 45, n. 2.

CARDOSO, Ruth Correa Leite, Recogido de una exposición realizada en el "Taller sobre las condiciones de vida de los sectores populares urbanos", realizado en el CEDES, Buenos Aires, del 4 al 7 de diciembre de 1979. Cualquier tergiversación de la exposición debe imputarse al autor de este trabajo.

CARLOS, Manuel L. y Lois SELLERS. Family, kinship structure and modernization en Latin America. En: LATIN AMERICAN RESEARCH REVIEW, Austin, University of Texas, v. VII, n. 2, Summer, 1972, pp. 95-124.

CARRASCO, Juan Carlos. Relación de pareja: un modelo analitico para el estudio de la fecundidad. Santiago, CELADE, noviembre de 1976. Serie A, n. 148.

CLAUSEWITZ, Carl von. De la guerra. Barcelona, Labor, 1976, 2a. ed.

CORSA, Leslie, Jr. and Deborah OAKLEY. Consequences of population growth for health services in less developed countries-an initial appraisal. En: National Academy of Sciences, Office of the Foreing Secretary: "RAPID POPULATION GROWTH, CONSEQUENCES AND POLICY IMPLICATIONS", v. II, Chapter X, pp. 368-402. Baltimore, The John Hopkins Press, 1972. Prepared by a Study Comittee of the Office of the Foreign Secretary, National Academy of Sciences, with the support of the Agency for International Development.

DI FILIPPO, Armando. El desarrollo económico y las teorias del valor. En: REVISTA DE LA CEPAL. Santiago, CEPAL,agosto de 1980, n. 11,pp. 81-119.

FILGUEIRA, Carlos. Expansión educacional y estratificación social en América Latina (19601970). Buenos Äires, Proyecto "Desarrollo y Educación en América Latina y el Caribe". 13 de septiembre de 1977. DEALC/4

FOUCAULT, Michel. Historia de la sexualidad. 1. La voluntad de saber. México, Siglo XXI, 1978.

FOX, Robin. Kinship and marriage. An anthropological perspective. Harmondsworth, Penguin books, 1967.

GARCIA BADARACCO, Jorge. La locura y la psiquiatria actual. En: LA NACION., Buenos Aires, domingo 13 de enero de 1980, Sección 4a. pág. 2 y domingo 20 de enero de 1980, Sección 4 a. pág. 2 .

GARCIA, Brígida, Humberto MUNOZ y Orlandina de OLIVEIRA. Hogar, trabajo y reposición de grupos sociales. Seminario sobre "Participación Femenina y Familia", Montevideo, 11-14 de diciembre de 1979. Citado como 1979. a.

GARCIA, Brígida, Humberto MUNOZ y Orlandina de OLIVEIRA. Hogar y fuerza de trabajo en la ciudad de México. Comunicación al"Taller sobre Estrategias de Supervivencia". Buenos Aires, 13 y 14 de marzo de 1980.

GARCIA, Brígida, Humberto MUNOZ y Orlandina de OLIVEIRA. Participación familiar en la actividad económica: México 1970. Seminario sobre "Participación Femenina y Familia", Montevideo, 11-14 de diciembre de 1979. Citado como 1979. b.

GARCIA, Brigida, Humberto MUNOZ y Orlandina de OLIVEIRA. Una caracterización so- 
ciodemográfica de las unidades domésticas en la ciudad de México. En: DEMOGRAFIA Y ECONOMIA, XIII, n. 1, v. 37, pp. 1-18.

GIFFIN, Karen. Familia, estrategias de supervivencia, fuerza de trabajo. Algunas consideraciones preliminares a una investigación sobre la población de Río de Janeiro. Rio de Janeiro, s.e., 1980. Comunicación al "Taller sobre Estrategias de Supervivencia". Buenos Aires, 13 y 14 de enero de 1980 .

GOMEZ, Sergio. Descomposición campesina: análisis de los asignatarios de la Reforma Agraria. Seminario "Dinámica de la economía campesina y el empleo en América Latina". PREAL/OIT, Grupo "Ocupación-desocupación/CLACSO". Santiago, 7 al 10 de enero de 1980.

GONZALEZ, Efrain. Economía campesina y empleo en el Cusco (Perú). Seminario "Dinámica de la economía campesina y el empleo en América Latina". PREALC/Ort. Grupo "Ocupación-desocupación"/CLACSO. Santiago, 7 al 10 de enero de 1980.

GOODE, William J. The theoretical importance of love. En: AMERICAN SOCIOLOGICAL REVIEW, v. 24, n. 1, February, 1959, pp. 38-47.

GRUPO DE ESTUDIOS AGRO-REGIONALES (GEA). Comunicación al Taller sobre "Estrategias de Supervivencia". Buenos Aires, 13 y 14 de marzo de 1980.

JOHNSON, Harry M. Sociología, una introducción sistemática. Buenos Aires, Paidós, 1965.

KOELLE, H. H. An experimental study on the determination of a definition for the "quality of li$f e$ ". En: REGIONAL STUDIES, Leicester, The Cavendish Press, v. 8, n. 1, March 1974, pp. $1-10$.

KNOX, P. L. Level of living: a conceptual framework for monitoring regional variation in wellbeing. En: REGIONAL STUDIES, Leicester, The Cavendish Press, v. 8, n. 1, March 1974, pp. 11-19.

LASCH, Christopher. The family and history. En: THE NEW YORK REVIEW, New York, November 13, November 27 and December 11, 1975.

LASLETT, Barbara. The family as a public and private institution: an historical perspective. En: JOURNAL OF MARRIAGE AND THE FAMILY, v. 35, n. 3, Agust 1973, pp. 480-492.

LIRA, Luis Felipe. Caracteristicas socioeconómicas y estructura de las familias en la ciudad de. Santiago, Chile, 1970. En: BURCH, Thomas K., Luis Felipe LIRA y Valdecir LOPES: LA FAMILIA COMO UNIDAD DE ESTUDIO DEMOGRAFICO. San José, CELADE, 1976, pp. 307-341. Citado como 1976. A.

LIRA, Luis Felipe. Introducción al estudio de la familia y el hogar. En: BURCH, Thomas K., Luis Felipe LIRA y Valdecir LOPES: LA FAMILIA COMO UNIDAD DE ESTUDIO DEMOGRAFICO. San José, CELADE, 1976, p. 5-46. Citado como 1976. b.

LOPES, Valdecir, La familia en el Brasil, según el censo de población de 1960. En: BURCH, Thomas K., Luis Felipe LIRA y Valdecir F. FLORES. LA FAMILIA COMO UNIDAD DE ESTUDIO DEMOGRAFICO. San José, CELADE, 1976, pp. 143-168.

LUXEMBURGO, Rosa. Introducción a la economía política. Córdoba, Pasado y Presente. 1972.

MARSHALL, Adriana. Notas sobre la determinación del salario. En: DESARROLLO ECONOMICO. Buenos Aires, n. 75, v. 19, octubre-diciembre, 1979, pp. 377-392.

MEILLASSOUX, Claude. Mujeres, graneros y capitales. Economía doméstica y capitalismo. México, Siglo XXI, 1979, 3a. ed.

MOLINA BARRIOS, Ramiro. Economía campesina y migracion: la venta estacional de fuerza de trabajo en el contorno rural de los centros mineros del norte de Potosí (Bolivia). Seminario "Dinámica de la economía campesina y el empleo en América Latina". PREALC/OIT. Grupo "Ocupación-desocupación/ClaCso. Santiago, 7 al 10 de enero de 1980.

MUELLBAUER, John. Household composition. Engel Curves and wellfare comparisons between households. $A$ duality approach. En: EUROPEAN ECONOMIC REVIEW, v. 5, n. 2, August 1974, pp. 103-122.

NACIONES UNIDAS. Consejo Económico y Social Comisión de Población. Informe del Simposio sobre la población y la familia. Honolulú, 6 al 15 de agosto de 1973, E/EN. 9/308, 19 de febrero de 1974.

NAG, Moni. Influence of socio-cultural, economic and demographic factors upon the family cycle. Influence of changes in the family cycle upon family life Socio-cultural patterns, family cycle and fertility. Symposium of Population and the Family, Honolulu, 6-15 August, 1973, E/CONF. 60/SYM. II/6. 22 June, 1973. 
NERLOVE, Marc. Economic growth and population: perspectives of the New Home Economics. En: JOURNAL OF POLITICAL ECONOMY, v. 82, n. 2, Part II, 1974.

NOUVEL OBSERVATEUR, LE, Et la famille, comment ca va? n. 626, du 8 au 14 novembre 1976, pp. 73-90. Et la famille, comment ça ira? n. 627, du 15 au 21 novembre, 1976, pp. 65-81.

OLIVEIRA, Maria Coleta F. A. de. Notas sobre união dos sexos e familia: tipos e fundamentos de legitimidade. Brasil, CEDIP, 1974, mimeo.

ORGANIZACION DE LOS ESTADOS AMERICANOS. Instituto interamericano de estadística. Programa del censo de América de 1970 (COTA-1970). Censos de habitación: temas, definiciones y clasificaciones utilizados por los paises de la región americana Washington, Secretaria General de la Organización de los Estados Americanos, 1977, cap. 9, Composición de los hogares, pp. 73-79..

PAIGE, Jelfrey M. Kinship and policy in stateless societies. En: AMERICAN JOURNAL OF SOCIOLOGY, v. 80, n. 2 September 1974, pp. 301-320.

PALAU VILADESAU. Tomás. Notas preliminares para el estudio de las estrategias familiares de supervivencia y el mercado de trabajo en el sector rural. Asunción, Centro Paraguayo de Estudios sociológicos, marzo, 1980.

PANTELIDES, Edith Alejandra. El hogar como unidad de análisis de los datos censales. importancia y posibilidades. EM: BURCH, Thomas K., Luis Felipe LIRA y Valdecir F. LOPES, "LA FAMILIA COMO UNIDAD DE ESTUDIO DEMOGRAFICO", San José CELADE, 1976, pp. 49-102.

PINTO, Aníbal y Armando DI FILIPPO. Desarrollo y pobreza en la América Latina: Un enfoque histórico-estructural". En: EL TRIMESTRE ECONOMICO, n. 183, julio-septiembre de 1979

RECCHINI DE LATTES, Zulma y Carlos A. BORSOTTI. Dos aspectos de las estrategias familiares: los tipos de hogares y su incidencia en la actividad económica femenina. A nálisis a partir de datos censales. Proyecto presentado a PISPAL, 1980.

ROUSSEL, Louis, Propositions pour una recherche "transversale" de démographie familiale, CICRED, 1980. "Une nouvelle facon d'aborder la coopération dans la recherche démographique", n. 2

SIEGEL. Jacob S. El hogar y la familia en la formulación de programas de vivienda, En: BURCH, Thomas K. Luis Felipe LIRA y Valdecir F. LOPES: "LA FAMILIA COMO UNIDAD DE ESTUDIO DEMOGRA FICO”. San José, CELADE, 1976, pp. 171-207.

SINGER, Paul. Leis de Populaçao e pesquisa de fertilidade, s. d. Citado como a.

SINGER, Paul. Comportamento reprodutivo e estrutura de classe, s. d., citado como b.

SUN TZU. El arte de la guerra. Buenos Aires, Sudamericana, 1973.

SUSSMAN, Marvin B. Issues and developments in family sociology in the 1970s. En: CURRENT RESEARCH IN SOCIOLOGY. August, 18-24, 1974, pp. 27-65.

TAEUBER, Irene. Modernization, population and the family: Cultural and historical perspectives. Change and transition in family structures. World Population Conference, 1974. Symposium on Population and the Family. Honolulu, 6-15 August, 1973. E/CONF. 60/SYM.II/5. 24 June, 1973.

UNITED NATIONS. Department of economic and social affairs. The determinants and consequences of population trends. New summary of findings on interaction of demographic, economic and social factors. New York, United Nations, 1973, ST/SOA/SER.A/50. Populations studies, n. 50, v. 1, chapter X, pp. 335-364. "Families and households".

WESTOFF, Charles F. La población y la familia: visión general. Bucarest, Conferencia Mundial de Población, 19 al 30 de agosto de 1974. E/CONF. 60/CBP/8, 18 de marzo de 1974. 